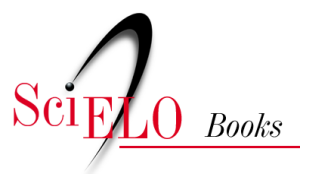

\title{
Capitulo 1 - Alimentação e nutrição na Atenção Básica: o cuidado como eixo estruturante
}

\author{
Ana Laura Brandão Motta \\ Ana Carolina Feldenheimer da Silva \\ Juliana Pereira Casemiro
}

\section{SciELO Books / SciELO Livros / SciELO Libros}

MOTTA, A. L. B., SILVA, A. C. F., and CASEMIRO, J. P. Alimentação e nutrição na Atenção Básica: o cuidado como eixo estruturante. In.: SILVA, A. C. F., MOTTA, A. L. B., and CASEMIRO, J. P., eds. Alimentação e nutrição na atenção básica: reflexões cotidianas e contribuições para prática do cuidado [onine]. Rio de Janeiro: EDUERJ, 2021, pp 11-34. ISBN: 978-65-87949-11-6. https://doi.org/10.7476/9786587949116.00002.

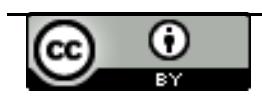

All the contents of this work, except where otherwise noted, is licensed under a Creative Commons Attribution 4.0 International license.

Todo o conteúdo deste trabalho, exceto quando houver ressalva, é publicado sob a licença Creative Commons Atribição 4.0.

Todo el contenido de esta obra, excepto donde se indique lo contrario, está bajo licencia de la licencia Creative Commons Reconocimento 4.0. 


\title{
Capitulo 1 \\ Alimentação e nutrição na Atenção Básica: \\ o cuidado como eixo estruturante
}

\author{
Ana Laura Brandão Motta \\ Ana Carolina Feldenheimer da Silva \\ Juliana Pereira Casemiro
}

Vários desenhos de Atenção Básica à Saúde foram experimentados no país desde a década de 1990, período em que se iniciou a utilização do termo Atenção Básica, em referência ao conjunto de ações no primeiro nível de atenção. A Atenção Básica $(\mathrm{AB})$ à Saúde, com especial deferência à Estratégia de Saúde da Família (ESF), pode ser considerada como uma das políticas federais de maior capilaridade e visibilidade do país e tem sido responsável pelo aumento do acesso de expressivo contingente populacional às açôes de saúde. ${ }^{1}$ Tal fato pode ser observado por meio do crescimento percentual de cobertura populacional da ESF nos últimos anos (de $17,43 \%$ da população brasileira coberta por ESF, em 2000, para 63,79\% de cobertura, em 2019). ${ }^{2}$

\footnotetext{
1 Vários autores discutem os significados dos termos Atenção Primária à Saúde (APS) e Atenção Básica em Saúde $(\mathrm{AB})$ e sua adequaçáo ao caso brasileiro. No Brasil, o Ministério da Saúde optou pelo uso do termo AB por conta da associação da APS ao enfoque restrito e seletivo predominante nas reformas latino-americanas, o que era incompatível com as diretrizes do Sistema Único de Saúde (Fausto, 2005; Castro e Machado, 2012). A Política Nacional de Atenção Básica de 2011 considera ambos os termos como equivalentes. No presente livro, optou-se por utilizar Atenção Primária à Saúde como conceito sinônimo de Atenção Básica, considerando-se o primeiro pela sua utilização internacional e o segundo em contexto nacional.

2 A partir da publicaçáo do Decreto N. o 9.795, de 17 de maio de 2019, a Atençáo Primária à Saúde (APS) no Brasil adquire status de Secretaria no âmbito do Ministério da
} 
A $A B$ exerce o papel de porta de entrada preferencial para o sistema de saúde, e suas unidades representam oportunidade de acesso regular de serviço por parte dos usuários. Além disso a $A B$ também exerce o papel de filtro em relação aos demais níveis de atenção, destacando sobretudo seu papel de condução, coordenação do processo do cuidado, integração com os outros níveis assistenciais e a regulação dos fluxos de atendimento (Giovanella et al., 2009).

A existência de um serviço de primeiro contato, procurado regularmente a cada vez que o usuário necessita de atençáo em caso de doença ou acompanhamento rotineiro, facilita, assim, a formação de vínculos e a coordenação dos cuidados na ESF, elementos importantes para a garantia da integralidade da atenção na $A B$. É nesse sentido que esse ponto da Rede de Atenção à Saúde (RAS) é destacado como fundamental na promoção da integralidade de atenção e do cuidado no Sistema Único de Saúde (SUS).

A expansão da cobertura da $\mathrm{AB}$ relaciona-se aos princípios doutrinários do SUS, por meio da universalização do acesso à saúde, da equidade e integralidade - observadas sobretudo nas décadas de 1990 e 2000 -, demarcam avanços importantes e (re)colocam no horizonte questóes que seguem sendo grandes desafios cotidianos dos serviços de saúde. Esse é o caso da efetivação de processos de trabalho coerentes com o princípio da integralidade (Faria e Araújo, 2010; Santos et al., 2018).

Mattos (2004) traz uma reflexáo importante sobre os sentidos da integralidade. Ele aponta três conjuntos de sentidos, a saber: o primeiro relaciona-se ao acesso universal a todos os níveis de cuidado; o segundo aborda a questáo da articulação entre assistência e prevenção; e, por fim, o terceiro sentido se relaciona à apreensão ampliada e prudente das necessidades de açôes e serviços de

Saúde, enfatizando-se, assim, sua relevância e prioridade no Sistema Único de Saúde (Brasil, 2019). 
saúde. Em especial, no último ponto, pode-se identificar a relação com a habilidade de reconhecer a adequação da oferta ao contexto específico da situação no qual se dá o encontro do sujeito com a equipe de saúde. Sob esse ponto de vista, um olhar mais crítico nos faz perceber grandes desafios, uma vez que esse modo de agir extrapola os referenciais do modelo biomédico, ainda hegemônico na formação e na prática cotidiana dos serviços de saúde. Por esse e por outros motivos, torna-se crescente a demanda por reflexóes mais sistemáticas sobre integralidade e cuidado. Estamos assim diante de uma longa jornada, uma vez que:

O tema cuidado e as noçôes dele derivadas não são originárias das ciências da saúde, embora aqui adquiram especificidades e, frequentemente, ainda se encontrem circunscritas ou aprisionadas aos sentidos mais operatórios ou técnicos do cuidar, do curar e do tratar (Sá et al., 2018, p. 26).

Observamos, sobretudo, que a noção do cuidado é polissêmica e transita por diferentes marcos conceituas e metodológicos. Ressaltamos a sensível descrição utilizada por Zoboli (2007, p. 63) sobre o cuidar como processo de "ir ao encontro do outro para acompanhá-lo e juntos promoverem e fomentarem a vida boa para todos. [...] é uma ação integral, com significados e sentidos que compreendem a saúde como 'direito de ser".

O cuidado se atualiza na concretude do cotidiano em saúde, em seus variados gestos. Por isso mesmo, sua superfície de desenvolvimento são fundamentalmente as práticas (Barros e Cesar, 2018). Estamos, então, num percurso que convida a valorizar e registrar passo a passo esse olhar mais amplo sobre a saúde e, de forma similar, sobre a alimentação e nutrição no contexto do SUS e da atenção básica.

Segundo a Política Nacional de Alimentação e Nutrição (PNAN), a atenção nutricional é compreendida como os cuidados 
relativos à alimentação e nutrição voltados à promoção e proteção da saúde, prevenção, diagnóstico e tratamento de agravos, que devem estar associados às demais ações de atenção à saúde do SUS, para indivíduos, famílias e comunidades, contribuindo para a conformação de uma rede integrada, resolutiva e humanizada de cuidados (Brasil, 2012). Nesse mesmo caminho, observa-se que o fortalecimento das perspectivas de autocuidado e de estratégias que contribuam para o apoderamento de informaçóes e construção de autonomia para escolhas alimentares mais saudáveis tornam-se aspectos cada vez mais presentes nos documentos oficiais (Brasil, 2012a; Brasil, 2014a), desafiando a construção de processos de trabalho mais cuidadosos.

Para os profissionais que trabalham na $\mathrm{AB}$, a prática do cuidado integral em alimentação e nutrição ganha materialidade tanto em âmbito individual (em consultas compartilhadas e procedimentos humanizados, entre outros) quanto em âmbito coletivo (em atividades de promoção a saúde e prevenção), assim como nas atividades que extrapolam os muros das Unidades Básicas de Saúde (UBS) e que recorrem à intersetorialidade como forma de buscar os sujeitos nas suas experiências de vida cotidiana no território, nas quais a cartografia das atividades dos sujeitos ajuda a equipe na compreensão do processo micropolítico em que o cuidado se dá, em ato (Carnut, 2017).

Fica evidente, assim, o quanto a Atenção Básica $(A B)$ é um espaço estratégico para o cuidado em alimentação e nutrição, em função de sua alta capilaridade e maior proximidade dos indivíduos e famílias em seu contexto social, apontando para o seu potencial de enfrentamento da situação epidemiológica por meio da consolidação de um modelo assistencial baseado nas necessidades de saúde da população e na integralidade do cuidado (Jaime et al., 2013).

A consolidação da ESF trouxe a necessidade de qualificação da Atenção Básica ofertada à população e, consequentemente, às ações de alimentação e nutrição que vêm sendo desenvolvidas ao longo dos anos. 
Este capítulo tem como objetivo apresentar uma reflexão que permita reconhecer os avanços relacionados ao cuidado em alimentação e nutrição na $\mathrm{AB}$, destacando os principais desafios para a garantia da integralidade do cuidado e para a qualificação dos processos assistenciais. Para tal, parte-se de análises que abarcam os principais aspectos normativos, análises relacionadas à estrutura das unidades básicas de saúde (UBS) e dos processos de trabalho desenvolvidos pelos profissionais da $\mathrm{AB}$.

\section{Avanços observados da alimentaçáo e nutrição no âmbito da Atençáo Básica}

Nas últimas décadas, podemos observar diferentes movimentos e iniciativas desenvolvidos pelo Ministério da Saúde (MS) que contribuíram para garantir e ampliar a organicidade da $A B$, sobretudo no que se refere à alimentação e nutriçáo.

Segundo Baptista (2007), os documentos formais expedidos pelo MS, por meio de sua consubstanciada edição de leis, diretrizes, portarias, atas e relatórios técnicos, são instrumentos importantes de regulação de políticas e visam a definir instruçôes para a execuçáo das leis, decretos e regulamentos aprovados pelo Poder Legislativo. A autora ressalta que, no contexto do setor de saúde, esses documentos têm assumido papel de grande destaque, tanto pelo quantitativo de documentos apresentados a partir dos últimos anos da década de 1990 quanto pelo forte poder de induçáo que esses instrumentos assumiram na definição da política.

Esse tipo de análise identifica, nas políticas e nos documentos oficiais, a pertinência em relação à situação do problema estudado, além de orientar a atuaçáo dos agentes governamentais no que tange aos atributos, à organização e à gestão dos serviços de saúde.

A seguir, o quadro 1 apresenta os principais marcos, documentos e políticas relacionados à alimentação e nutrição na Atenção Básica nas últimas décadas. 
Quadro 1. Descrição dos principais marcos, documentos e políticas relacionados à alimentação e nutrição na Atenção Básica entre os anos de 1999 e 2019

\begin{tabular}{|c|c|}
\hline Ano & Ação \\
\hline 1999 & $\begin{array}{l}\text { Primeira edição da Política Nacional de Alimentação e Nutrição (PNAN) } \\
\text { (Brasil, 1999). }\end{array}$ \\
\hline 2002 & $\begin{array}{l}\text { Dez Passos para uma alimentação saudável: guia alimentar para crianças menores } \\
\text { de } 2 \text { anos: um guia para o profissional da saúde na Atençâo Básica (Brasil, 2002). }\end{array}$ \\
\hline \multirow{2}{*}{2005} & Programa Nacional de Suplementação de Vitamina A (Brasil, 2005). \\
\hline & Programa Nacional de Suplementação de Ferro (Brasil, 2005a). \\
\hline \multirow{4}{*}{2006} & Política Nacional de Promoção da Saúde (Brasil, 2006). \\
\hline & Primeira edição da Política Nacional de Atenção Básica (Brasil, 2006a). \\
\hline & $\begin{array}{l}\text { Primeira edição do Guia alimentar para a população brasileira: promovendo a } \\
\text { alimentação saudável (Brasil, 2006b). }\end{array}$ \\
\hline & $\begin{array}{l}\text { Portaria Interministerial n. }{ }^{\circ} 1010 \text {, de } 8 \text { de maio de 2006: Institui as diretrizes para } \\
\text { a Promoção da Alimentação Saudável nas Escolas de educação infantil, fundamen- } \\
\text { tal e nível médio das redes públicas e privadas, em âmbito nacional (Brasil, 2006c). }\end{array}$ \\
\hline \multirow{2}{*}{2007} & Programa Saúde na Escola (PSE) (Brasil, 2007). \\
\hline & Caderno de Atenção Básica (n. 20): "Carências de micronutrientes" (Brasil, 2007a) \\
\hline 2008 & $\begin{array}{l}\text { Portaria do Ministério da Saúde n.o } 154 \text { de } 24 \text { de janeiro de 2008: Cria os Núcleos } \\
\text { de Apoio à Saúde da Família - NASF (Brasil, 2008). }\end{array}$ \\
\hline 2009 & Rede de Nutrição do Sistema Único de Saúde (RedeNutri). \\
\hline 2011 & Programa Academia da Saúde (Brasil, 2011). \\
\hline \multirow[b]{2}{*}{2012} & Segunda edição da Política Nacional de Atenção Básica (Brasil, 2012a). \\
\hline & $\begin{array}{l}\text { Marco de Referência de Educação Alimentar e Nutricional para as Políticas Públi- } \\
\text { cas (Brasil, 2012b). }\end{array}$ \\
\hline \multirow[t]{2}{*}{2013} & $\begin{array}{l}\text { Estratégia Nacional para Promoção do Aleitamento Materno e Alimentação Com- } \\
\text { plementar Saudável no SUS - Estratégia Amamenta e Alimenta Brasil (Brasil, } \\
\text { 2013). }\end{array}$ \\
\hline & Segunda edição da Política Nacional de Alimentação e Nutrição (Brasil, 2013a). \\
\hline
\end{tabular}




\begin{tabular}{|c|c|}
\hline \multirow{5}{*}{2014} & Reformulação do Programa Nacional de Suplementação de Ferro (Brasil, 2014). \\
\hline & Segunda edição do Guia Alimentar para a População Brasileira (Brasil, 2014 a). \\
\hline & $\begin{array}{l}\text { Cadernos de Atenção Básica (n. 38): "Estratégias para cuidado da pessoa com } \\
\text { doença crônica obesidade" (Brasil, 2014b). }\end{array}$ \\
\hline & Segunda edição da Política Nacional de Promoção da Saúde (Brasil, 2014c). \\
\hline & $\begin{array}{l}\text { Cadernos de Atenção Básica (n. 39): "Núcleo de Apoio à Saúde da Família - } \\
\text { Volume 1: Ferramentas para a gestão e para o trabalho cotidiano" (Brasil, 2014d). }\end{array}$ \\
\hline \multirow{3}{*}{2015} & $\begin{array}{l}\text { Estratégia de fortificação da alimentação infantil com micronutrientes em pó - } \\
\text { NutriSUS (Brasil, 2015). }\end{array}$ \\
\hline & $\begin{array}{l}\text { Cadernos de Atenção Básica (n. 23): "Saúde da Criança: Aleitamento Materno e } \\
\text { Alimentação Complementar" (Brasil, 2015a). }\end{array}$ \\
\hline & $\begin{array}{l}\text { Caderno de atenção domiciliar, v. } 3 \text { - "Cuidados em Terapia Nutricional" (Brasil, } \\
\text { 2015b). }\end{array}$ \\
\hline \multirow{2}{*}{2017} & $\begin{array}{l}\text { Publicação: Contribuições dos Núcleos de Apoio à Saúde da Família para a Atenção } \\
\text { Nutricional (Brasil, 2017). }\end{array}$ \\
\hline & Terceira edição da Política Nacional de Atenção Básica (Brasil, 2017a). \\
\hline 2018 & $\begin{array}{l}\text { Portaria n. }{ }^{\circ} 2.706 \text {, de } 18 \text { de outubro de } 2017 \text { : Criação do Programa crescer sau- } \\
\text { dável - Programa de prevenção, controle e tratamento da Obesidade Infantil } \\
\text { (Brasil, 2018a). }\end{array}$ \\
\hline \multirow[t]{2}{*}{2019} & $\begin{array}{l}\text { Decreto N. 9.795, de } 17 \text { de maio de } 2019 \text { - Atenção Primária à Saúde (APS) } \\
\text { adquire status de Secretaria no âmbito do Ministério da Saúde (Brasil, 2019). }\end{array}$ \\
\hline & Segunda edição do Guia Alimentar para crianças menores de 2 anos (Brasil, 2019a). \\
\hline
\end{tabular}

Fonte: Elaboração própria.

Por trás do conjunto de publicaçóes apresentados no quadro 1, destacam-se avanços e mudanças de concepçóes sobre a nutrição e o cuidado nutricional expressos em sua forma teórica, mas objetivando alterar as práticas das equipes de saúde. Foi um processo gradual de mudanças que ocorreu de forma articulada à criação e consolidação do conceito de Rede de Atenção à Saúde. Por outro lado, os debates da Segurança Alimentar e Nutricional (SAN) influenciaram decisivamente grande parte dos marcos legais e incidiram de forma importante para revisão de programas, açôes e políticas de saúde e nutrição, e na relação com diversos setores. Dentre as mudanças apresentadas, pode-se destacar o fortalecimento da perspectiva da alimentaçáo enquanto direito que tem 
influenciado abordagens mais integrais e integradas do tema alimentação e nutrição nos documentos oficiais, chamando a atenção para inclusão dos aspectos relacionados à comensalidade, cultura e diversidade alimentar. A consolidação de um conceito de "alimentação adequada e saudável" deu-se nos anos de 2006/2007 a partir de amplo debate no Conselho Nacional de Segurança Alimentar e Nutricional (CONSEA, 2007) e passou a integrar documentos oficiais de diversos setores, inclusive do setor da saúde. Compreende-se alimentação adequada e saudável como:

prática alimentar apropriada aos aspectos biológicos e socioculturais dos indivíduos, bem como ao uso sustentável do meio ambiente. Deve estar de acordo com as necessidades de cada fase do curso da vida e com as necessidades alimentares especiais; ser referenciada pela cultura alimentar e pelas dimensões de gênero, raça e etnia; ser acessível do ponto de vista físico e financeiro; harmônica em quantidade e qualidade; baseada em práticas produtivas adequadas e sustentáveis; com quantidades mínimas de contaminantes físicos, químicos e biológicos (Brasil, 2012).

Com esse conceito, a já consolidada ideia de "alimentação saudável" incorporou-se à noção de "alimentação adequada", ainda pouco popularizada, mas fundamental para o reforço da noção de direito humano à alimentação (Paiva et al., 2019). Partir dessa referência, que amplia a noção de alimentação, abrem-se inúmeras possibilidades de qualificação das perspectivas de cuidado e autocuidado a serem desenvolvidos e aperfeiçoados na $A B$.

Ao lado da SAN, outro aspecto que impactou sobremaneira o repensar o campo da alimentação e nutrição foi a incorporação dos referenciais da Promoção da Saúde (Ferreira e Magalhães, 2007). Ao considerar a saúde como prática socialmente construída, compreendida em um enfoque institucional e estratégico que integra os contextos dos atores sociais, a Promoção da Saúde tem sido 
apontada como importante resposta aos desafios no que tange às questôes de alimentação e nutrição.

É relevante destacar que, uma $\mathrm{AB}$ ampliada e mais robusta pressupóe uma preocupação com as estratégias de Promoção da Saúde e com os determinantes sociais da saúde e doença, demandando açóes intersetoriais que promovam a ampliaçáo das iniciativas de cuidado que envolvam a população e outros setores.

Destaca-se, como exemplo, nesse sentido, o fortalecimento da articulação intersetorial, principalmente com o Ministério da Educação - por meio da inter-relação com programas, como o Programa Saúde na Escola - e com o Ministério do Desenvolvimento Social e Agrário - principalmente no acompanhamento que envolve as condicionalidades do Programa Bolsa Família (Castro e Machado, 2012).

Nesse mesmo caminho, e aprimorando as formas de exercitar a intersetorialidade, a Câmara Interministerial de Segurança Alimentar e Nutricional (CAISAN) representou importante lócus de articulação e tomada de decisão em políticas públicas. Iniciativas como a Estratégia Intersetorial de Prevenção e Controle da Obesidade (Brasil, 2014e) e do Pacto para Alimentação Saudável (Brasil, 2015e) são exemplos importantes dessa afirmação. Além disso, documentos como o Plano de Açóes Estratégicas para o Enfrentamento das Doenças Crônicas Não Transmissíveis (DCNT) no Brasil, 2011-2022 (Brasil, 2011b); Marco de Referência para Educação Alimentar e Nutricional nas Políticas Públicas (Brasil, 2012b), a segunda edição do Guia Alimentar para a População Brasileira (Brasil, 2014a), a segunda edição da publicação Alimentos Regionais Brasileiros (Brasil, 2015c) e o Guia Alimentar para crianças brasileiras menores de 2 anos (Brasil, 2019b), entre outros, são expressôes dessa atualização da abordagem que considera o debate intersetorial na definição de estratégias e que reforçam a perspectiva da Soberania e Segurança Alimentar e Nutricional. 
Propostas inovadoras e ousadas foram engendradas para o campo da alimentação e nutrição, com apostas importantes na $\mathrm{AB}$ como espaço privilegiado para se tornar concreto. Implícito a essa aposta está o pressuposto de uma RAS fortalecida e de Núcleos Ampliados de Saúde da Família (NASF) estruturados e vinculados a equipes adequadamente instaladas nos diferentes territórios. Destaca-se, assim, a relevância da criação dos NASFs em 2008, constituídos por equipes compostas por profissionais de diferentes áreas para atuarem na lógica de apoio matricial, em parceria com a ESF, compartilhando as práticas em saúde nos territórios sob a sua responsabilidade (Castro e Machado, 2012).

Entende-se que estas são conquistas importantes nesse campo, contudo, cabe considerar que há frequentemente um grande hiato entre a mudança de conceitos, bem como as perspectivas criadas em políticas públicas, e sua real efetivação no nível local. Outra questão importante é considerar que o contexto local nem sempre é receptivo e favorável a mudanças tão profundas como as que foram propostas nas últimas décadas. Dessa forma, destacam-se como elementos estratégicos, nesse contexto, as iniciativas que possam fortalecer capacidades no nível local para o desenvolvimento de processos continuados de avaliação, monitoramento e planejamento no âmbito das ações de alimentação e nutrição na $\mathrm{AB}$.

\section{A estrutura das UBS e sua relaçáo com o cuidado em alimen- taçáo e nutriçáo}

Reconhecendo os avanços relacionados aos marcos legais descritos acima, cabe ressaltar uma questão fundamental: para a efetivação do adequado cuidado em alimentação e nutrição aos usuários, é necessário que exista suficiente disponibilidade de estrutura.

No campo da avaliação, a estrutura é um dos componentes para a análise de desempenho e resolutividade dos serviços de saúde, considerando que estruturas adequadas favorecem a oferta 
de serviços oportunos e de qualidade (Donabedian, 1988). Nessa mesma direção, o referido autor propôs um esquema sistêmico de análise que considera a tríade: estrutura, processo e resultado.

A estrutura refere-se às condiçóes relativamente estáveis dos serviços de saúde, entre elas: os instrumentos, os recursos materiais e humanos e o contexto físico e organizacional no qual são realizadas as açôes de saúde. Dessa forma, as características da estrutura contribuem para o desenvolvimento dos processos de cuidado e influenciam seus consequentes resultados. Os indicadores de estrutura revelam as condiçóes estruturais para a melhoria e qualidade da prestação dos serviços, todavia, esta não seria uma relação causal de certeza, mas de probabilidade.

Nesse caminho, os dados do Programa Nacional de Melhoria do Acesso e da Qualidade da Atenção Básica (PMAQ-AB) representam um potente recurso para análise. Esse Programa foi criado em 2011 pelo MS e tem como principal objetivo induzir a ampliaçáo do acesso e a melhoria da qualidade da Atenção Básica por meio da instituiçáo de processos contínuos e progressivos que ampliem a capacidade das três esferas de governo em ofertar serviços com garantia de um padrão de qualidade comparável nacional, regional e localmente (Brasil, 2011a).

O PMAQ-AB investiga o desempenho das equipes por um conjunto de critérios e está organizado em fases que se complementam e que conformam um ciclo contínuo de melhoria do acesso e da qualidade da $A B$. As equipes de $A B$ aderiram voluntariamente ao PMAQ- $\mathrm{AB}$ e contemplaram primordialmente três eixos de avaliação: estrutura, processo e satisfação do usuário (Cruz et al., 2014).

As informaçóes disponibilizadas pelo PMAQ permitem monitorar o cuidado na $\mathrm{AB}$ sobre as mais diferentes perspectivas. Aqui, focaremos naquelas que têm potencialidades para o campo da alimentação e nutrição.

Para traçar algumas reflexões sobre esse ponto, foram analisados os dados da avaliação externa do ciclo 2 do PMAQ-AB 
(realizado entre novembro de 2013 e abril de 2014), com base nas variáveis correspondentes às ações de alimentação e nutrição.

Tabela 1. Disponibilidade de equipamentos nas Unidades Básicas de Saúde no Brasil (Brasil, 2019)

\begin{tabular}{lll}
\hline Equipamento & $\%$ & $\mathbf{N}$ \\
\hline $\begin{array}{l}\text { Balança antropométrica de } \\
150 \mathrm{~kg}\end{array}$ & 83,4 & 20062 \\
\hline $\begin{array}{l}\text { Balança antropométrica de } \\
200 \mathrm{~kg}\end{array}$ & 23,14 & 5568 \\
\hline Balança infantil & 92,9 & 22351 \\
\hline Régua antropométrica & 88,9 & 21392 \\
\hline Fita métrica & 96,6 & 23232 \\
\hline
\end{tabular}

Fonte: Elaboração própria a partir dos dados do ciclo 2 do PMAQ-AB. Ciclo disponibilizado pela coordenação do PMAQ-AB.

Os dados apresentados na tabela 1 demonstram que a evolução nos marcos normativos apresentados no item anterior foi acompanhada de expressiva melhoria no que se refere à garantia de disponibilidade de estrutura - compreendida como instalaçóes, equipamentos e insumos - destinada às açóes de alimentação e nutrição nas UBS.

Quanto à presença de equipamentos para realização da Vigilância Alimentar e Nutricional (VAN) nas unidades de AB, identificou-se que mais de $80 \%$ das Unidades apresentam balanças para adultos, crianças, régua antropométrica e fita métrica.

Entende-se como VAN o monitoramento das condiçóes de alimentação e nutrição de uma determinada população. Destaca-se a relevância de que essa ação seja contínua e sistemática, de modo a permitir identificação dos casos, estratificação de risco e organização da oferta de cuidado. Sua execução deve estar associada às demandas espontâneas e programadas das UBS (Brasil, 2014b; Brasil, 2015d). A avaliação tomou por base equipamentos relacionados à VAN, 
levando em conta que tais açóes foram historicamente priorizadas e que constituem elemento estruturante do cuidado em alimentação e nutrição na $\mathrm{AB}$.

A adequada disponibilidade de equipamentos pode estar relacionada à necessidade de realização de avaliação antropométrica para acompanhamento realizado por grandes programas nacionais, tais como, o Programa Bolsa Família (PBF), o Sistema de Vigilância Alimentar e Nutricional (SISVAN) e o HIPERDIA, um programa que se destina ao cadastramento e acompanhamento de portadores de hipertensão arterial e/ou diabetes mellitus atendidos (Brasil, 2013b; Brasil, 2013c).

Contudo, um elemento preocupante é que menos de um quarto das unidades possuem balança com capacidade entre 150 e $200 \mathrm{~kg}$, o que apresenta uma barreira para o diagnóstico e tratamento das pessoas com obesidade. Considerando os alarmantes dados relacionados ao sobrepeso e obesidade no Brasil e compreendendo o papel da $\mathrm{AB}$ como coordenadora do cuidado, pode-se destacar que esse dado releva uma fragilidade em termos de disponibilidade de estrutura.

\section{Cuidado em alimentaçáo e nutriçáo: o que dizem os dados do PMAQ-AB sobre os processos de trabalho?}

Ao lado dos avanços relacionados aos marcos legais e dos ganhos de estrutura para o cuidado em alimentação na $\mathrm{AB}$, deve-se destacar o desafio da adequação dos processos de trabalho, elemento que decisivamente está relacionado à qualidade das práticas de cuidado ofertadas.

Entende-se como processos de trabalho em saúde as atividades relacionadas à produção e consumo de serviços dessa área - envolvendo usuários, tecnologias e recursos humanos. Em última análise, estamos diante de expressóes do cotidiano dos serviços de saúde e da atuação de trabalhadores/profissionais de saúde (Pereira, 2008). 
Nesse olhar para o cotidiano, deve-se observar que o trabalho em saúde, sobretudo na $\mathrm{AB}$, apresenta algumas particularidades. Destacam Faria e Araújo (2010) que o trabalho em saúde é complexo, dinâmico e criativo e se realiza no espaço de negociação entre o trabalho prescrito (normas, tempo para operação, ferramentas utilizadas) e o trabalho real (o que acontece, ainda que as condiçóes de realização não sejam as idealizadas). Nesse mesmo caminho, Santos et al. (2018, p. 862) destacam a ESF como "campo de possibilidades", em que o trabalho em saúde pautado pela integralidade se materializa em "práticas direcionadas às necessidades objetivas e subjetivas das pessoas em seu contexto social, apreendidas e transformadas em açôes por uma equipe multiprofissional".

Assim, reconhecendo que os debates acerca dos processos de trabalho em saúde têm se tornado mais frequentes e que abarcam dimensóes objetivas e, sobretudo, subjetivas complexas, cabe destacar que, neste capítulo, travamos breve aproximação a partir do PMAQ-AB. Foram selecionados dados relativos à atuação do NASF e dos processos de trabalho relacionados à obesidade. Considerou-se para essa escolha a importância deste agravo uma vez que mais da metade da populaçáo brasileira encontra-se com excesso de peso (IBGE, 2010; Brasil, 2018) e que esse fato tem impactado os serviços, representando um dos maiores desafios para as açóes do cuidado em saúde, em especial as ações de alimentação e nutrição.

A tabela 2 apresenta os dados do PMAQ-AB referentes ao processo de trabalho relacionado a essa temática. Pouco mais da metade das equipes de Atenção Básica no Brasil ofertam atividades em grupo para pessoas com obesidade. Apenas um terço das equipes fazem o registro e controle das pessoas com obesidade sob sua responsabilidade, percentual semelhante encontrado no que se refere ao registro de agendamento e encaminhamento de usuários a partir da classificação do risco. Menos de metade das equipes ofertam consultas para tratar a obesidade e menos de $40 \%$ possuem registro dos usuários encaminhados à Atenção Especializada. 
Tal fato deixa claro uma fragilidade das equipes de $A B$ no que diz respeito à coordenação do cuidado e ordenaçáo do percurso terapêutico dos indivíduos nas RAS, impactando, assim, o acesso aos distintos níveis de atenção e comprometendo o percurso assistencial e a resolutividade do cuidado na $\mathrm{AB}$.

Tabela 2. Processos de trabalho relacionados à obesidade desenvolvidos no âmbito da Atenção Básica no Brasil (Brasil, 2019)

\begin{tabular}{|c|c|c|}
\hline Atividade & $\%$ & $\mathbf{N}$ \\
\hline $\begin{array}{l}\text { Para quais grupos a equipe oferta açóes? } \\
\text { Pessoas com obesidade }\end{array}$ & 57,1 & 17010 \\
\hline $\begin{array}{l}\text { A equipe possui o registro do seu território? } \\
\text { Das pessoas com obesidade }\end{array}$ & 36,4 & 10832 \\
\hline $\begin{array}{l}\text { A equipe programa oferta de consultas para quais } \\
\text { situaçôes? } \\
\text { Obesidade }\end{array}$ & 46,3 & 13782 \\
\hline $\begin{array}{l}\text { A programaçáo da agenda da equipe está de acordo com } \\
\text { o risco classificado para quais situaçôes? } \\
\text { Obesidade }\end{array}$ & 35,9 & 10678 \\
\hline $\begin{array}{l}\text { A equipe mantém registro dos usuários de maior risco } \\
\text { encaminhados para outros pontos de atenção? } \\
\text { Obesidade }\end{array}$ & 39,0 & 11608 \\
\hline
\end{tabular}

Fonte: Elaboração própria a partir dos dados do ciclo 2 do PMAQ-AB. Ciclo disponibilizado pela coordenação do PMAQ-AB.

Os dados apresentados revelam grande descompasso com as açóes preconizadas. Para a organização da atenção para prevenção e controle da obesidade, espera-se que a $\mathrm{AB}$ seja capaz de: realizar vigilância alimentar e nutricional prevendo que a mensuraçáo de dados seja realizada de forma oportuna e capaz de subsidiar classificação de risco; adequado acolhimento e captaçáo a partir de profissionais de saúde "capacitados e sensibilizados para a temática"; e o apoio diagnóstico e terapêutico, o que exige, além das questôes de infraestrutura da UBS, oferta e acesso a exames solicitados e a medicamentos, oferta de práticas integrativas e complementares, apoio matricial e articulação com os demais pontos da RAS (Brasil, 2014b). 
Logo, os dados apresentados deixam aparente a fragilidade dos processos de trabalho. Os baixos registros sobre a obesidade e encaminhamento dos usuários com obesidade demonstram que a capacidade de coordenaçáo do cuidado e a da promoção da longitudinalidade do cuidado, atributos da $\mathrm{AB}$, estáo prejudicadas. Por seu turno, a baixa realizaçáo de grupos prejudica estratégias importantes, uma vez que abrir espaços de produção de diálogo, reflexão e problematização junto à comunidade possibilita a construção de relação de corresponsabilidade, favorecendo formas mais humanas e efetivas do processo de trabalho em saúde. A constituição de grupos operativos e outras estratégias coletivas que oportunizem fortalecimento do autocuidado são atividades inerentes à $\mathrm{AB}$ (Brasil, 2014b).

$\mathrm{O}$ acolhimento representa outro atributo fundamental à $\mathrm{AB}$, visto que representa dispositivo potente para atender à exigência de acesso, além de propiciar vínculo entre equipe e população, trabalhador e usuário. Desdobra-se dessa afirmação uma reflexão importante: que elementos relacionados ao cuidado em alimentação e nutrição devem ser aprimorados em relação às açôes de acolhimento?

Outro ponto importante é a valorização do apoio matricial como dispositivo para organizar o trabalho interprofissional, tanto em equipes - como as de NASF - quanto em redes de atenção à saúde. Pode-se afirmar que o aumento da cobertura da ESF reflete diretamente na ampliação de escopo das açóes, corroborada principalmente pelo crescente número de equipes do NASF, que passaram de 1.317, em 2000, para 4.406, em 2016 (Brasil, 2016), suscitando, assim, a necessidade da análise do trabalho desenvolvido por essas equipes.

No detalhamento das açôes do NASF, verificamos que mais de $70 \%$ das equipes realizam atividades de Atençáo Nutricional e desenvolvem açôes de promoção da alimentação adequada e saudável - açôes voltadas para os agravos relacionados à alimentação $\mathrm{e}$ nutrição e vigilância alimentar e nutricional. A açáo menos prati- 
cada é a articulação intersetorial com a SAN, por cerca de $60 \%$ dos Núcleos, o que aponta para uma necessidade de discussão das ações da equipe de NASF para fora dos muros das unidades de saúde.

Tabela 3. Ações de alimentação e nutrição desenvolvidas pelos Núcleos de Apoio à Saúde da Família no Brasil (Brasil, 2017)

Atividade $\%$

O NASF apoia o desenvolvimento de açóes de promoção da alimentação adequada e saudável

73,7

O NASF apoia o desenvolvimento de açóes voltadas para os agravos relacionados à alimentação e nutrição

73,1

O NASF apoia o desenvolvimento de açóes de vigilância alimentar e nutricional

O NASF apoia o desenvolvimento de açóes de articulação intersetorial para garantia da SAN

60,1

Fonte: Elaboração própria a partir dos dados do ciclo 2 do PMAQ-AB. Ciclo disponibilizado pela coordenação do PMAQ-AB.

$\mathrm{Na}$ avaliação realizada com as equipes de NASF, foram questionados os temas que as equipes haviam escolhido para trabalhar nas açóes da AB (tabela 4). A "Atenção Nutricional” foi citada por $73,8 \%$ das equipes, seguida do "Apoio à Organização do Processo de Trabalho das Equipes" citado por 43,9\% das equipes. Outros temas citados foram: "Saúde do Trabalhador" (24,9\%), "Assistência Farmacêutica" (23,6\%), "Práticas Integrativas e Complementares" $(22,4 \%)$, revelando o fato de que a "Atenção Nutricional" se configura como um tema recorrente de grande parte das equipes, seja esse fato um reflexo da presença do nutricionistas nas equipes ou uma preocupação crescente por conta do aumento do excesso de peso, hipertensão e diabetes na população atendida pelo SUS. 
Tabela 4. Percentual dos temas escolhidos pelas equipes de NASF para ser trabalhado na Atenção Básica no Brasil (Brasil, 2017)

\begin{tabular}{lc}
\hline Temas & $\%$ \\
\hline Atenção nutricional & 73,8 \\
\hline Apoio à organização do processo de trabalho das equipes & 43,9 \\
\hline Saúde do trabalhador & 24,9 \\
\hline Assistência farmacêutica & 23,6 \\
\hline Práticas integrativas e complementares & 22,4 \\
\hline
\end{tabular}

* Cada equipe NASF pode selecionar até dois temas.

Fonte: Elaboração própria a partir dos dados do ciclo 2 do PMAQ-AB. Ciclo disponibilizado pela coordenação do PMAQ-AB.

O lugar de destaque dado ao tema da Atenção Nutricional dentro das equipes NASF revela, por um lado, uma forte conexão com o peso da prevalência da obesidade/sobrepeso e das doenças relacionadas à nutriçáo nos serviços de $\mathrm{AB}$ e, por outro, o reconhecimento da promoção de práticas alimentares saudáveis como estratégia fundamental para ações de prevenção e promoção da saúde.

Pode-se, a título de horizonte e objeto em construção, pensar que a Atenção Nutricional está justamente na interface possível com processos de trabalho que se organizam a partir do cuidado como ato singular de encontros: "as redes começam onde há desejo ou vontade de cuidar” (Franco, 2015, p. 261).

\section{Consideraçóes finais}

O recente histórico de publicação de marcos legais e documentos orientadores relacionados à alimentação e nutrição demonstra um fortalecimento desse campo no âmbito das políticas públicas de saúde. Coerente com esse fenômeno, identifica-se, no bojo dos 
exitosos esforços para qualificar e ampliar a estrutura nas UBS, que foram incorporados itens afetos às ações de alimentação e nutrição.

Apesar dos avanços em estrutura, nenhum dos itens apresentados alcançou $100 \%$ de adequação e, portanto, merecem acompanhamento contínuo e novos investimentos. Assim, a utilização de dados do PMAQ-AB faz pensar sobre suas múltiplas possibilidades de utilização para apoiar a gestão nas três esferas de governo, incluindo a gestão local e sobretudo as Áreas Técnicas de Alimentaçáo e Nutrição. Fortalecer capacidades no campo da avaliação, monitoramento e planejamento em alimentação e nutrição é estratégia fundamental para ampliar a qualidade das açóes na $A B$ e outros pontos da RAS.

Como grande desafio, aponta-se a necessidade de fortalecimento das estratégias de reorganização dos processos de trabalho no âmbito da ESF a partir da valorização do trabalho em equipe, dos esforços para qualificar a relaçáo entre trabalhador/profissional de saúde e usuário e para o fortalecimento das estratégias de acolhimento. Esses são pontos-chave para promover mudanças nos processos de produção do cuidado. Além disso, é imperativo reafirmar a premissa do atendimento a todas as pessoas que procuram os serviços de saúde, garantindo o acesso à $\mathrm{AB}$, assim como aos demais pontos da RAS para alcance da integralidade.

Iniciativas de educação permanente que visem a estimular o desenvolvimento da consciência nos profissionais sobre o seu contexto e suas responsabilidades acerca das práticas de cuidado em alimentação e nutrição representam relevante estratégia para a qualificação de processos de trabalho. Contudo, é necessário destacar que outros elementos estáo relacionados à melhoria dos processos de trabalho na $\mathrm{AB}$ e incluem questôes como: remuneração, tipo de vínculo, valorização da carreira e outros aspectos que interferem nas condiçóes de trabalho e, por sua vez, nas condiçôes de cuidar.

Serão necessárias novas reflexôes sobre as açōes da alimentação e nutrição, bem como para a identificação de caminhos para 
qualificar os processos de cuidado. Com a organização deste capítulo, buscou-se apresentar um panorama procurando abrir espaços, sinalizar lacunas e apontar alguns horizontes nesta longa travessia que, esperamos, possa nos conduzir a um tempo-espaço de ações mais cuidadosas no campo da alimentação e nutrição na Atenção Básica à Saúde, pautadas na interdisciplinaridade, integralidade, intersetorialidade e, sobretudo, na valorização da autonomia e da alteridade.

\section{Referências}

BAPTISTA, T. W. F. "Análise das portarias ministeriais da saúde e reflexões sobre a condução nacional da política de saúde". Caderno de Saúde Pública, v. 23, n. 3, pp. 615-26, 2007.

BARROS, M. E. B. e CESAR, J. M. "A Saúde em práticas de cuidado: dialogia e cogestão". In SÁ, M. C. et al. Organização do cuidado e práticas em saúde. Rio de Janeiro. Editora: Fiocruz, 2018.

BRASIL. Ministério da Saúde. Politica Nacional de Alimentação e Nutrição. Brasília: Ministério da Saúde, 1999.

. Ministério da Saúde. Dez Passos para uma alimentação saudável: guia alimentar para crianças menores de 2 anos: um guia para o profissional da saúde na Atenção Básica. Brasília: Ministério da Saúde, 2002.

. Ministério da Saúde. Portaria n. ${ }^{\circ}$ 729, de 13 de maio de 2005. Institui o Programa Nacional de Suplementação de Vitamina A e dá outras providências. Lex: Diário Oficial da União, 2005.

. Ministério da Saúde. Portaria n. ${ }^{\circ} 730$, de 13 de maio de 2005. Institui o Programa Nacional de Suplementação de Ferro, destinado a prevenir a anemia ferropriva e dá outras providências. Lex: Diário Oficial da União, 2005a.

. Ministério da Saúde. Secretaria de Vigilância em Saúde. Política nacional de promoção da saúde. Brasília: Ministério da Saúde, 2006.

. Ministério da Saúde. Secretaria de Atenção à Saúde. Departamento de Atenção Básica. Política Nacional de Atenção Básica. Brasília: Ministério da Saúde, 2006a.

- Ministério da Saúde. Guia alimentar para a população brasileira: promovendo a alimentação saudável. Brasília: Ministério da Saúde, 2006b.

. Portaria Interministerial n. ${ }^{\circ} 1.010$, de 8 de maio de 2006. Institui as diretrizes para a Promoção da Alimentação Saudável nas Escolas de educação infantil, 
fundamental e nivel médio das redes públicas e privadas, em âmbito nacional. Lex: Diário Oficial da União, 2006c.

. Presidência da República. Casa Civil. Subchefia para Assuntos Jurídicos. Decreto n. ${ }^{\circ}$ 6.286, de 5 de dezembro de 2007. Institui o Programa Saúde na Escola - PSE, e dá outras providências. Lex: Diário Oficial da União, 2007.

. Ministério da Saúde. Carências e micronutrientes. Brasília: Ministério da Saúde, 2007a. (Cadernos de Atenção Básica, n. 20. Série A. Normas e Manuais Técnicos).

. Ministério da Saúde. Portaria n. ${ }^{\circ} 154$, de 24 de janeiro de 2008. Cria os Núcleos de Apoio à Saúde da Família - NASF. Orientaçóes para a implantação dos núcleos. Lex: Diário Oficial da União, 2008.

. Ministério da Saúde. Portaria n. ${ }^{\circ} 719$, de 7 de abril de 2011. Institui o Programa Academia da Saúde no âmbito do Sistema Único de Saúde. Brasília: Ministério da Saúde, 2011.

. Ministério da Saúde. Portaria n. ${ }^{\circ} 1.654$, de 19 de julho de 2011. Institui, no âmbito do Sistema Único de Saúde, o Programa Nacional de Melhoria do Acesso e da Qualidade da Atenção Básica (PMAQ-AB) e o Incentivo Financeiro do PMAQ-AB, denominado Componente de Qualidade do Piso de Atenção Básica Variável - PAB Variável. Lex: Diário Oficial da União, 2011a.

. Ministério da Saúde. Secretaria de Vigilância em Saúde. Plano de ações estratégicas para o enfrentamento das doenças crônicas não transmissiveis (DCNT) no Brasil 2011-2022. Brasília: Ministério da Saúde, 2011b.

. Ministério da Saúde. Política Nacional de Alimentação e Nutrição. 2 ed. Brasília: Ministério da Saúde, 2012.

. Ministério da Saúde. Portaria n. ${ }^{\circ} .488$, de 21 de outubro de 2011. Aprova a Politica Nacional de Atenção Básica, estabelecendo a revisão de diretrizes e normas para a organização da Atenção Básica, para a Estratégia Saúde da Familia (ESF) e o Programa de Agentes Comunitários de Saúde (PACS). Brasília: Ministério da Saúde, 2012.

- Ministério do Desenvolvimento Social e Combate à Fome. Marco de referência de educação alimentar e nutricional para as políticas públicas. Brasília: Ministério de Desenvolvimento Social e Combate à Fome, 2012a.

. Ministério da Saúde. Nota técnica 26/2013. Institui a Estratégia Nacional para Promoção do Aleitamento Materno e Alimentação Complementar Saudável no Sistema Único de Saúde (SUS) - Estratégia Amamenta e Alimenta Brasil. Brasília: Ministério da Saúde, 2013.

. Ministério da Saúde. Secretaria de Atenção à Saúde. Departamento de Atenção Básica. Política Nacional de Alimentação e Nutrição. Brasília: Ministério da Saúde, 2013a. 
. Ministério da Saúde. Secretaria de Atenção à Saúde. Departamento de Atenção Básica. Estratégias para o cuidado da pessoa com doença crônica: diabetes mellitus. Brasília: Ministério da Saúde, 2013b.

. Ministério da Saúde. Secretaria de Atenção à Saúde. Departamento de Atenção Básica. Estratégias para o cuidado da pessoa com doença crônica: hipertensão arterial sistêmica. Brasília: Ministério da Saúde, 2013c.

. Ministério da Saúde. Gabinete do Ministro. Portaria n. ${ }^{\circ}$ 1.977, de 12 de setembro de 2014. Atualiza as diretrizes nacionais do Programa Nacional de Suplementação de Ferro (PNSF) da Politica Nacional de Alimentação e Nutrição (PNAN). Lex: Diário Oficial da União, 2014.

. Ministério da Saúde. Guia alimentar para a população brasileira. Brasília: Ministério da Saúde, 2014a.

- Ministério da Saúde. Secretaria de Atenção à Saúde. Departamento de Atenção Básica. Estratégias para o cuidado da pessoa com doença crônica: obesidade. Brasília: Ministério da Saúde, 2014b.

. Ministério da Saúde. Portaria n. ${ }^{\circ}$ 2.446, de 11 de novembro de 2014. Redefine a Politica Nacional de Promoção da Saúde (PNPS). Lex: Diário Oficial da União, 2014c.

- Ministério da Saúde. Núcleo de Apoio à Saúde da Família, volume 1. Brasília: Ministério da Saúde, 2014d. (Cadernos de Atenção Básica, n. 39).

. CAISAN. Estratégia Intersetorial de Prevenção e Controle da Obesidade. Brasília: CAISAN 2014e.

. Ministério da Saúde. Estratégia de fortificação da alimentação infantil com micronutrientes em pó. Brasília, DF, 2015. Disponível em: http://dab.saude.gov. $\mathrm{br} /$ portaldab/pse.php?conteudo=nutrisus. Acesso em 28/05/2017

- Ministério da Saúde. Secretaria de Atenção à Saúde. Departamento de Atenção Básica. Saúde da criança: aleitamento materno e alimentação. 2 ed. Brasília: Ministério da Saúde, 2015a.

. Ministério da Saúde. Secretaria de Atenção à Saúde. Departamento de Atenção Básica. Caderno de atenção domiciliar - Volume 3 - Cuidados em Terapia Nutricional. Brasília: Ministério da Saúde, 2015b.

- Ministério da Saúde. Secretaria de Atenção à Saúde. Departamento de Atenção Básica. Alimentos regionais brasileiros. Brasília: Ministério da Saúde, 2015c.

. Ministério da Saúde. Secretaria de Atenção à Saúde. Departamento de Atenção Básica. Marco de Referência para Vigilância Alimentar e Nutricional na Atenção Básica. Brasília: Ministério da Saúde, 2015d.

. Decreto n. ${ }^{\circ} 8.553$, de 3 de novembro de 2015. Institui o Pacto Nacional para Alimentação Saudável. Brasília, 2015e. 
- Ministério da Saúde. Secretaria de Atenção à Saúde. Departamento de Atenção Básica. Contribuições dos Núcleos de Apoio à Saúde da Família para a Atenção Nutricional. Brasília: Ministério da Saúde, 2017.

. Ministério da Saúde. Portaria n. ${ }^{\circ} 2.436$, de 21 de setembro de 2017. Aprova a Politica Nacional de Atenção Básica, estabelecendo a revisão de diretrizes para a organização da Atenção Básica, no âmbito do Sistema Único de Saúde (SUS). Brasília: Ministério da Saúde, 2017a.

. Ministério da Saúde. Secretaria de Vigilância em Saúde. Departamento de Vigilância de Doenças e Agravos Não Transmissíveis e Promoção da Saúde. Vigitel Brasil 2017: vigilância de fatores de risco e proteção para doenças crônicas por inquérito telefônico: estimativas sobre frequência e distribuição sociodemográfica de fatores de risco e proteção para doenças crônicas nas capitais dos 26 estados brasileiros e no Distrito Federal em 2017. Brasília: Ministério da Saúde, 2018. Disponível em: http://bvsms.saude.gov.br/bvs/publicacoes/vigitel_brasil_2017_vigilancia_fatores_riscos.pdf. Acesso em 20 de novembro 2018.

. Portaria n. ${ }^{\circ}$ 2.706, de 18 de outubro de 2017. Lista os Municipios que finalizaram a adesão ao Programa Saúde na Escola para o ciclo 2017/2018 e os habilita ao recebimento do teto de recursos financeiros pactuados em Termo de Compromisso e repassa recursos financeiros para Municípios prioritários para açôes de prevenção da obesidade infantil com escolares. Brasília, 2018a.

. Decreto n. ${ }^{\circ}$ 9.795, de 17 de maio de 2019. Aprova a Estrutura Regimental e o Quadro Demonstrativo dos cargos em comissão e das funçôes de confiança do Ministério da Saúde, remaneja cargos em comissão e funções de confiança, transforma funções de confiança e substitui cargos em comissão do Grupo-Direção e Assessoramento Superiores - DAS por Funçôes Comissionadas do Poder Executivo - FCPE. Brasília: 2019.

. Decreto n. ${ }^{\circ} 8.553$, em 03 de novembro de 2015. Institui o Pacto Nacional para Alimentação Saudável. Brasília: CAISAN, 2019a.

- Ministério da Saúde. Secretaria de Atenção Primária. Departamento de Promoção da Saúde. Guia alimentar para crianças brasileiras menores de 2 anos. Brasília: Ministério da Saúde, 2019b.

CARNUT, L. "Cuidado, integralidade e atenção primária: articulação essencial para refletir sobre o setor saúde no Brasil”. Saúde em Debate, v. 41, n. 115, pp. 1177-86, Rio de Janeiro, 2017.

CASTRO, A. L. B., e MACHADO, C. V. "A política federal de Atenção Básica à saúde no Brasil nos anos 2000". Physis: revista de saúde coletiva, v. 22, n. 2, 2012.

CRUZ, M. M. D. et al. "Uses of the planning and self-evaluation in the working processes of Family Health teams in Primary Care”. Saúde em Debate, v. 38, n. spe, pp. 124-39, 2014. 
DONABEDIAN, A. "The quality of care. How can it be assessed?" Journal of the American Medical Association, 260 ed., pp. 1743-8, 1988.

FARIA, H. X. e ARAUJO, M. D. "Uma perspectiva de análise sobre o processo de trabalho em saúde: produção do cuidado e produção de sujeitos". Saúde e Sociedade, v. 19, n. 2, pp.429-39, 2010.

FAUSTO, M. C. R. Dos programas de medicina comunitária ao Sistema Único de Saúde: uma análise histórica da atenção primária na politica de saúde brasileira (Tese). Universidade do Estado do Rio de Janeiro, 2005.

FERREIRA, V e MAGALHÃES, R. "Nutrição e promoção da saúde: perspectivas atuais”. Cadernos de Saúde Pública, v. 23, n. 7, pp. 1674-81, 2007.

FRANCO, T. B. "Redes de cuidado: conexão e fluxo para o bom encontro com a saúde”. ALMEIDA, P. F. et al. (orgs.). Atenção Primária à Saúde na coordenação do cuidado em Regiōes de Saúde. Salvador: EDUFBA, 2015, pp. 89-113.

GIOVANELLA, L. et al. "Saúde da família: limites e possibilidades para uma abordagem integral de atenção primária à saúde no Brasil”. Ciência \& Saúde Coletiva, v. 14, n. 3, pp. 783-94, 2009.

IBGE. Pesquisa de Orçamentos Familiares 2008-2009 - POF. Rio de Janeiro, 2010.

JAIME P. C. et al. "Brazilian obesity prevention and control initiatives". Obesity Reviews, v. 14, n. s2, pp. 88-95, 2013. Disponível em: https://onlinelibrary. wiley.com/doi/epdf/10.1111/obr.12101. Acesso em 11 dez. 2018.

MATTOS, R. A. A. "A integralidade na prática (ou sobre a prática da integralidade)". Cadernos de Saúde Pública, v. 20, n. 5, pp. 1411-6, 2004.

PAIVA, J. B. et al. "A confluência entre o 'adequado' e o 'saudável': análise da instituição da noção de alimentação adequada e saudável nas políticas públicas do Brasil". Cadernos de Saúde Pública, v. 35, n. 8, 2019.

PEREIRA, I. B. e LIMA, J. C. F. Dicionário da educação profissional em saúde. Rio de Janeiro: EPSJV, 2008.

SÁ, M. C. et al. "Apresentação". In et al. Organização do cuidado epráticas em saúde. Rio de Janeiro. Editora: Fiocruz, 2018.

SANTOS, D. S. et al. "Processos de trabalho na Estratégia de Saúde da Família: potencialidades da subjetividade do cuidado para reconfiguração do modelo de atenção”. Ciência \& Saúde Coletiva, v. 23, n. 3, pp. 861-70, 2018.

ZABOLI, E. "Cuidado: práxis responsável de uma cidadania moral". In PINHEIRO, R e MATTOS, R. A. Razóes públicas para a integralidade em saúde: o cuidado como valor. Rio de Janeiro: IMS/UERJ: CEPESC: ABRASCO, 2007. 